\title{
Research of on-line noise source identification based on the Grey neural network
}

\author{
Yang Yang and Shuangwei Wang \\ School of Physics, NENU-Northeast Normal University, 130024, Changchun, China
}

\author{
Nailan Hao \\ Changchun Medical Higher Training School, 130031, Changchun, China
}

\author{
Yan Zhu, Yanan Tian and Shengdan Li \\ School of Physics, NENU-Northeast Normal University, 130024, Changchun, China
}

\begin{abstract}
(Received 18 December 2007; accepted 22 August 2008)
The accurate identification of an acoustic source is a fundamental problem in noise control. In real measurements, if a contribution of separate sources to the whole is identified properly, it is possible to apply appropriate modifications to the sound source that contributes most significantly to the whole signal. This paper presents a new approach to acoustic noise identification by using modern spectrum estimation, Grey Relational Analysis (GRA), and Back-Propagation neural networks. The modern spectrum estimation method was used to recognize the main noise source, and GRA was used to estimate the similarity among different curves of the power spectrum. Finally, the Artificial Neural Network (ANN) was used to do curve fitting, which give the results more wide applications. The ranking of the noise sources was determined on the basis of their individual contributions to the overall noise. The results of simulations confirmed the feasibility and validity of the method proposed in this paper, and it will play an important role in noise control, signal source identification, and other fields.
\end{abstract}

\section{INTRODUCTION}

Noise has received increasing recognition as one of our critical environmental pollution problems besides air and water pollution. Noise and vibration measurements are is of great importance for noise detection, environmental protection, labour protection, etc. The best way to control noise is at an adequate sound source. This approach decreases the complexity of the work and helps people research new products that generate undesired sound at a lower level. ${ }^{1}$

According to reference ${ }^{2}$, if the main noise source can be correctly identified, then it is possible to decide which machines and which parts of the hall should be treated. The treatment may consist of partially or completely enclosing a machine, in inserting acoustic screens between the source and the place to protect, or in placing absorbing materials on the corresponding wall, etc.

However, for an existing multi-source noise, the exact locations of the primary noise sources are difficult to recognize by the human ear due to the remarkable accumulation effect. ${ }^{3}$ For a multi-source noise, an effective method of noise control is to find the main source, which contributes the most noise to the total. So we need to know the specific weight of each noise source compared to the whole at the given place. In general, noise signals are very complex and are composed of noises produced by different sources. The reason is that the sound field, at any point, is a combination of the various contributions from all actual acoustic sources and reflections associated with walls and other obstacles in a given room. ${ }^{2}$ To get a reliable sample of the actual noise, measurements should be carried out while the machines are constantly in operation. Therefore, a suitable method aimed at accurate noise source identification must be proposed.

The identification of the number and locations of acoustic sources is a fundamental problem in noise control. This subject of identifying acoustic sources has been investigated extensively in the past, and some general methods have been proposed as well.

Reference $^{4}$ describes active noise control (ANC) systems. These systems have received a lot of attention in recent years. The ANC system uses the principle of acoustic superposition to attenuate an undesired sound. It involves an electro-acoustic system generating a signal with an opposite phase that cancels the unwanted noise. It has to be adaptive because of changes in the environment, degradation of system components, and alterations in the noise source itself.

Czyzewski ${ }^{5}$ described a computer learning system based on neural network, which indicates the propagating direction and locations of the primary sound source. However, the values of the sound pressure level with respect to individual sound sources are not available. Lan et $\mathrm{al}^{3}$ use the method of genetic algorithms (GA) in determining the best locations and SWLs (sound power levels) of noises by minimizing the overall square of the variation. The results reveal that the accuracy of noise recognition decreases when the number of primary noise sources increases. Furthermore, the time required for noise source identification increases considerably when the number of monitoring points is increased. ${ }^{3}$ 